\title{
Regnase-1, a Ribonuclease Involved in the Regulation of Immune Responses
}

\author{
SHIZUO AKIRA ${ }^{1,2,3}$ \\ ${ }^{1}$ Laboratory of Host Defense, World Premier Institute Immunology Frontier Research Center, \\ Osaka University, 3-1 Yamada-oka, Suita, Osaka 565-0871, Japan \\ ${ }^{2}$ Department of Host Defense, Research Institute for Microbial Diseases (RIMD), Osaka \\ University, 3-1 Yamada-oka, Suita, Osaka 565-0871, Japan \\ ${ }^{3}$ Correspondence: sakira@biken.osaka-u.ac.jp
}

\begin{abstract}
Zc3h12a is a lipopolysaccharide-inducible gene and has a CCCH-type zinc-finger domain. Zc3h12a knockout mice develop spontaneous autoimmune diseases accompanied by splenomegaly and by lymphadenopathy. Subsequent studies show that $\mathrm{Zc} 3 \mathrm{~h} 12 \mathrm{a}$ is a nuclease involved in destabilization of interleukin (IL)- 6 and IL-12 mRNA via the stem loop structure within the $3^{\prime}$ UTR of these genes. Thus, we renamed it regulatory RNase-1 (Regnase-1) based on its function. ІкB kinases phosphorylate and degrade Regnase-1 protein in macrophages in response to IL-1R/Toll-like receptor (TLR) stimulation. T-cell-specific deletion of Regnase-1 produces pathogenic T cells with hyperactivated phenotypes as well as autoimmune diseases. Regnase1 protein is cleaved by Malt1 and inactivated in response to T-cell receptor stimulation. Thus, Regnase-1 is a key molecule in the regulation of immune responses.
\end{abstract}

Immune responses are key host reactions against microorganisms and foreign substances that protect our bodies from their harmful effects. The immune response must be promptly initiated, be sufficiently strong to eradicate pathogens, and be rapidly terminated properly to avoid tissue damage and pathological inflammation as well as autoimmunity. Excessive or prolonged immune responses are characterized by overproduction of proinflammatory cytokines such as tumor necrosis factor (TNF), interleukin-1 (IL-1), and IL-6. These cytokines are also involved in tissue damage by chronic inflammation as well as the development of autoimmune diseases. Dynamic changes of cytokine expression are controlled by the regulatory circuits of positive transcription factors $\mathrm{NF}-\kappa \mathrm{B}, \mathrm{C} / \mathrm{EBPs}$, and AP-1 family and negative factors such as ATF3. Thus far, changes in gene expression have been considered mostly as a result of transcriptional control. However, accumulating recent evidence suggests that the immune system uses posttranscriptional regulation, particularly for the expression of cytokine and chemokine genes that show the most dynamic changes of expression (Anderson 2010; Ivanov and Anderson 2013). mRNAs of these genes are enriched in AU-rich elements (AREs) in their $3^{\prime}$ untranslated regions (UTRs). The conservation of an adenylate/uridylate (AU)-rich region was observed in genes encoding cytokines, which are inflammatory mediators. Shaw and Kamen (1986) presented the first direct evidence that ARE function as potent mRNA-destabilizing elements by showing that a conserved region of 51 nucleotides containing AUUUA motifs from the $3^{\prime}$ UTR of human granulocyte-macrophage-colony-stimulating factor (GM-CSF) mRNA degraded $\beta$-globin mRNA when inserted into the $3^{\prime}$ UTR of $\beta$-globin mRNA, which is otherwise stable. AU-rich sequences bearing AUUUA motifs from c-fos, c-myc, nur77, junB, interferon (IFN)- $\beta$, and IL-3 mRNA also function as RNA destabilizing elements. Subsequent bioinformatic analyses estimated that $5 \%-8 \%$ of the transcriptome contains AREs (Bakheet et al. 2006) that serve as binding sites for a number of regulatory proteins that either destabilize or stabilize mRNA. ARE-directed mRNA degradation is influenced by many exogenous factors, including phorbol esters, calcium ionophores, and cytokines. Several kinase pathways regulate the activity of ARE-binding proteins, and thereby coordinate the expression of their target mRNAs. More than half of the transcriptomic changes that occur during T-cell activation are regulated at the level of mRNA stability and are not accompanied by transcriptional changes (Cheadle et al. 2005). Recently, Hao and Baltimore (2009) showed that intrinsic mRNA stability is particularly important for the expression of cytokine and chemokine genes that show the most dynamic expression patterns. mRNAs of these genes are enriched for AREs in the 3' UTRs confirming the prominent role of AREs in mRNA stability.

\section{DEADENYLATION-DEPENDENT AND -INDEPENDENT mRNA DECAY}

There are two types of mRNA degradation pathways: deadenylation-dependent and deadenylation-independent mRNA decay (Schoenberg and Maquat 2012). Deadenylation-dependent mRNA decay is the major pathway in mammalian cells. The first step in this decay is polyA tail removal. Following deadenylation, mRNA degradation continues in a $5^{\prime}-3^{\prime}$ or $3^{\prime}-5^{\prime}$ direction. $5^{\prime}-3^{\prime}$ mRNA 
decay begins with decapping. Nonstop mRNA decay and nonsense-mediated mRNA decays are mRNA surveillance pathways for eliminating aberrant mRNAs. In contrast to normal mRNAs with termination codons, mRNAs that lack termination codons are recognized and degraded by a mechanism called nonstop mRNA decay. The nonstop decay pathway releases ribosomes that have reached the far $3^{\prime}$ end of an mRNA and guides mRNA to the exosome complex for degradation. The cap structure can be removed independently of the deadenylation step in the case of mRNAs containing a premature termination codon. This pathway is called nonsense-mediated mRNA decay. Endonuclease cleavage of mRNAs also occurs without prior deadenylation. Even though endonuclease cleavage was one of the first identified mechanisms of mRNA decay, until recently this was considered a minor process that acted on a specific part of the transcriptome or functioned in parallel with the more prevalent processes of deadenylation and exonuclease-catalyzed decay (Schoenberg 2011).

Tristetraprolin (TTP) is the most well characterized and extensively studied ARE-binding protein (Brooks and Blackshear 2013). TTP is the founding member of a small family of $\mathrm{CCCH}$ tandem zinc-finger proteins. TTP was initially cloned as an immediate-early response gene induced by tetradecanoyl phorbol acetate (TPA) in murine fibroblasts, which was named TPA-induced sequence 11 (TIS11). The TTP family consists of TTP (ZFP36), ZFP36L1, ZFP36L2, and ZFP36L3. All members bind to AREs, and are characterized by the presence of a particular tandem zinc-finger domain (TZF) in their coding sequence. TTP was named after the particular repeated motif (PPPPG) of the protein. TTP was initially characterized as a key inflammation-induced Tnf mRNA-destabilizing factor whose deficiency resulted in multiple chronic inflammatory syndromes including arthritis, cachexia, and dermatitis in mice. Figure 1 shows the mechanism of mRNA degradation by TTP proteins. Deg- radation of mRNAs by TTP is one example of deadenylation-dependent mRNA decay. mRNA has a looped conformation mediated by interactions between eukaryotic initiation factor 4 and poly-A-binding proteins. Binding of TTP to ARE triggers destabilization of circularized mRNA, which induces the recruitment of deadenylases and subsequent removal of the cap structure by decapping enzyme and finally degradation of the mRNA body by exosome and exonucleases.

\section{TOLL-LIKE RECEPTOR (TLR) FAMILY: FUNCTION AND SIGNALING}

Toll was initially identified in insects as a receptor essential for dorsoventral polarity during embryogenesis. In 1996, Jules Hoffmann's group revealed its essential role in the innate immune response of insects to fungal infection (Lemaitre et al. 1996). This finding led to the identification of Toll homologs in mammals. Thus far, more than 10 members of the TLR family have been identified in mammals. TLR comprises a large superfamily with IL-1R members based on similarities in their cytoplasmic regions. TLRs and IL-1R family members share a conserved stretch of $\sim 200$ amino acids in their cytoplasmic region known as the "TLR and IL-1R-related" (TIR) domain. The extracellular portion of TLRs comprises leucine-rich repeats (LRR), whereas that of IL-1R is three immunoglobulin domains. Individual TLR members recognize different microbial components and trigger inflammatory and immune responses (Fig. 2) (Beutler 2009; Iwasaki and Medzhitov 2010; Takeuchi and Akira 2010).

Similarly, the gene expression patterns among TLR members are also diverse by means of activation of different intrasignaling pathways (O’Neill and Bowie 2007; Kawai and Akira 2010). The difference in TLR signaling pathways are in part due to selective usage of adaptor molecules, MyD88 and TRIF (Fig. 2). MyD88 is

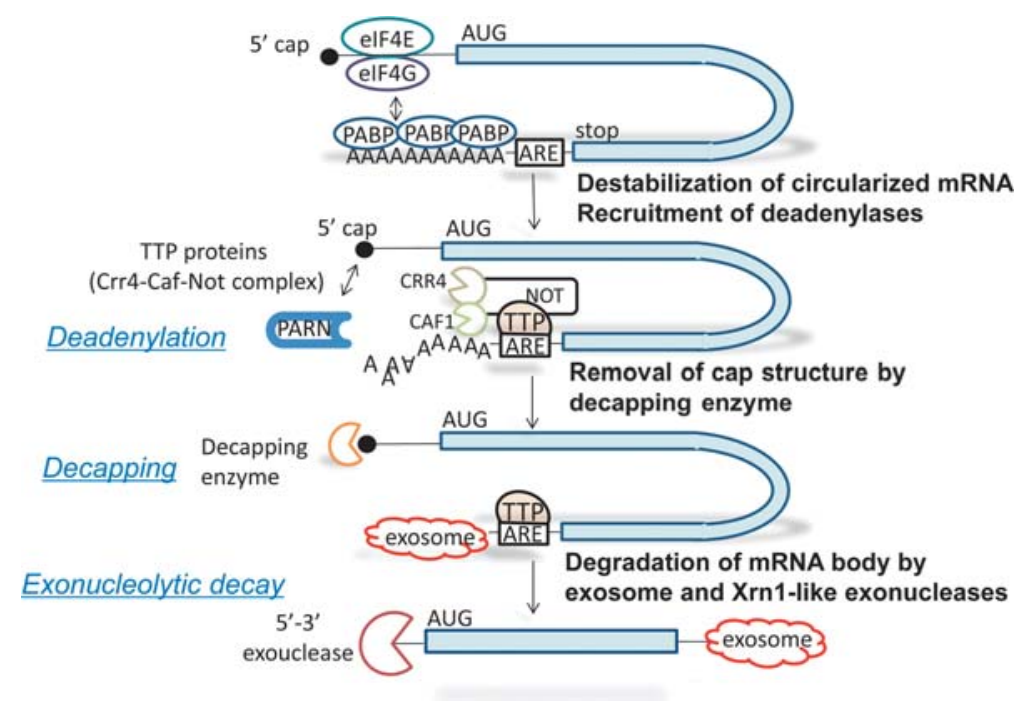

Figure 1. Involvement of TTP in mRNA degradation. 


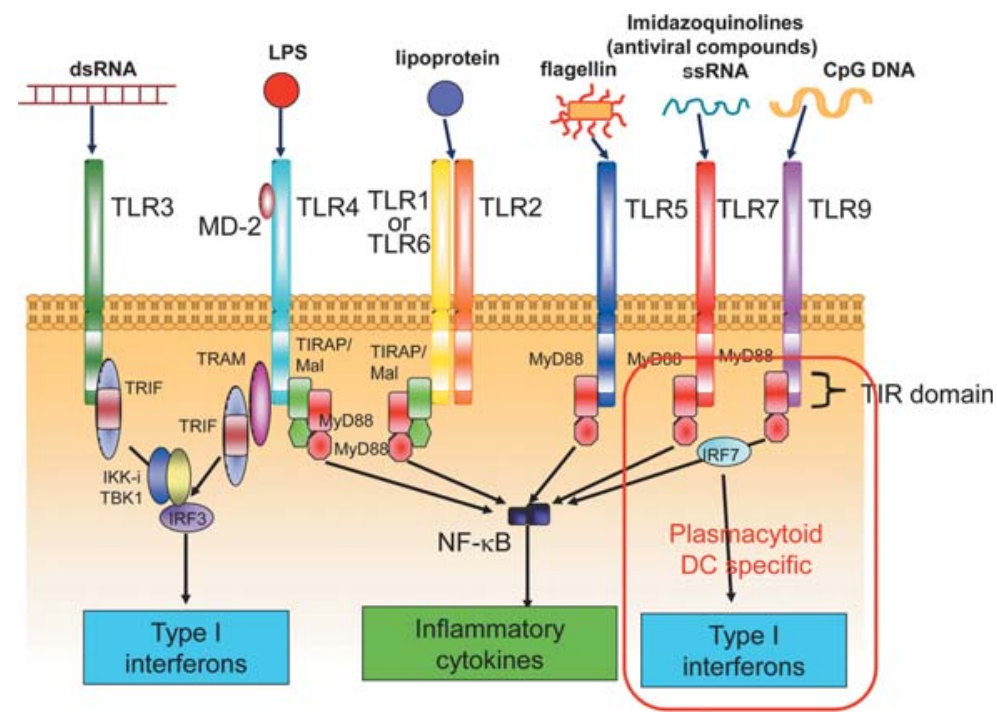

Figure 2. TLRs: ligands and signaling pathways. Individual TLRs recognize different microbial components. For example, TLR3 recognizes double-stranded RNA and, therefore, is a detector of viral invasion in the body. TLR4 recognizes LPS, a cell wall component of Gram-negative bacteria. TLR2 recognizes peptidoglycan, lipoteichoic acid, and lipoproteins, all of which are cell wall components of Gram-positive bacteria. TLR5 recognizes flagellin, a major component of bacterial flagella, and is responsible for the detection of intestinal pathogen invasion. TLR7 recognizes antiviral synthetic compounds imidazoquinolines, and single-stranded RNA as a natural ligand. TLR9 recognizes bacterial and viral unmethylated CpG DNA. Similarly, signaling pathways of TLR are different from each other. The difference in signaling pathways among TLRs is in part due to the selective usage of adaptor molecules. MyD88 is essential for all TLR signaling pathways except TLR3 signaling. TRIF is responsible for type-1 IFN production in TLR3 and TLR4 signaling. TLR7 and TLR9 induce type-1 IFN by a MyD88-dependent mechanism in plasmacytoid dendritic cells.

responsible for signaling mediated by all TLRs except for TLR3 and is involved in inflammatory responses. In response to TLR ligands, IL-1R-associated kinase 4 (IRAK4) associates with MyD88 and activates IRAK1 and IRAK2. The IRAKs then dissociate from MyD88 and interact with TRAF6, an E3 ubiquitin protein ligase, along with other E2 ubiquitin protein ligases, which result in activation of a complex containing transforming growth factor (TGF)- $\beta$-activated kinase 1 (TAK1), TAK1-binding protein (TAB1), TAB2, and TAB3. Activation of the TAK1/TAB complex triggers both the MAPK and NF-кB signaling pathways. IKK- $\beta$, along with IKK- $\alpha$ and NEMO (IKK- $\gamma$ ), forms the IKK complex that mediates the phosphorylation of $\mathrm{I} \kappa \mathrm{B} \alpha$, a NF- $\mathrm{\kappa B}$ inhibitory protein that masks the nuclear localization signal

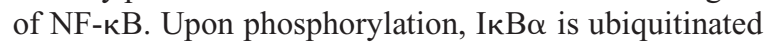
and degraded, freeing NF-KB to translocate into the nucleus and initiate the transcription of inflammatory cytokines.

Besides the MyD88-dependent pathway, a MyD88-independent pathway exists in TLR3 and TLR4 signaling. This pathway is triggered by another adaptor molecule, TRIF, and is involved in antiviral responses by producing type-1 IFN. TRIF associates with TRAF3 and TRAF6 through a binding domain present on its amino terminus. The activation of TRAF6 and RIP1 by TRIF leads to NF- $\kappa \mathrm{B}$ activation. TRAF3 mediates IRF3 activation via activation of TBK1 and IKKi, noncanonical IKKs. The phosphorylation of IRF3 by TBK1/IKKi leads to dimer formation and nuclear translocation of IRF3 and the induction of IFN- $\beta$. TLR7 and TLR9 also induce type 1
IFN. In this case, the mechanism of type1 IFN induction is different from that induced by TLR3 and TLR4. MyD88 directly binds IRF7, and after undergoing phosphorylation, IRF7 translocates from the cytoplasm to the nucleus and produces type 1 IFN, particularly IFN- $\alpha$. This pathway is activated only in plasmacytoid dendritic cells in contrast to TRIF-dependent type 1 IFN induction detected in every cell type, and is responsible for a huge amount of type 1 IFN produced in response to systemic viral infection.

\section{Zc3h12a, AN EARLY LIPOPOLYSACCHARIDE (LPS) INDUCIBLE GENE, IS AN ENDORIBONUCLEASE}

To date, many TLR ligands have been identified and TLR signaling pathways have been clarified. Our research direction is now moving toward the effector phase of TLR responses - that is, to find out the role of genes rapidly induced in response to TLR ligands. To examine TLR-induced gene expression comprehensively, we performed microarray analysis using mouse macrophages from wild-type, MyD88 knockout (KO), and TRIF KO mice stimulated with LPS, a TLR4 ligand. When we identified LPS-inducible genes whose functions were unknown and that contained interesting motifs in the amino acid sequence, we generated their $\mathrm{KO}$ mice and examined their role. Among them, we recently identified a functionally unknown gene named Zc3h12a. Zc3h12a has a $\mathrm{CCCH}$-type zinc-finger motif, and forms a family with $\mathrm{Zc} 3 \mathrm{hb}$, c, and d. Northern blot analysis showed that 
Zc3h12a mRNA is rapidly induced in mouse macrophages in a MyD88-dependent manner after LPS stimulation and gradually decreased with time.

MCP-1 (CCL2) is the main chemotactic factor for migration of monocytes/macrophages and is involved in the pathogenesis of chronic inflammation. Zc3h12a was initially reported as the most highly induced mRNA by MCP-1, and therefore it was named MIP-1 inducible protein 1 (MIPIP1) (Zhou et al. 2006). Cardiomyocyte-targeted expression of MCP-1 in mice caused heart failure, accompanied by increased expression of MCPIP1, implying a role of MCPIP-1 in cardiomyocyte cell death. Later, the existence of MCPIP family members, MCPIP1, 2, 3, and 4 were reported and the expression of MCPIP1 and MCPIP3 were shown to be highly induced in macrophages in response to LPS (Liang et al. 2008a, b). Forced expression of MCPIP1 inhibited LPS-induced TNF- $\alpha$ and nitric oxide production as well as their gene expression, suggesting a role of MCPIP1 as a negative regulator in macrophage activation. Previously, MCP-1-induced angiogenesis was shown to be mediated via MCPIP, at least in part, through transcriptional activation of cadherins 12 and 19 (Niu et al. 2008). Taken together, these studies describe MCPIP1 as a transcription factor. However, fraction experiments performed in our laboratory revealed that $\mathrm{Zc} 3 \mathrm{~h} 12 \mathrm{a}$ protein is mainly localized in the cytoplasm.

To examine the functional role of Zc3h12a, we generated Zc3h12a KO mice (Matsushita et al. 2009). Zc3hc12a KO mice were born alive at a Mendelian ratio, but most of them died spontaneously within $12 \mathrm{wk}$ after birth. Zc3h12a KO mice showed severe splenomegaly and lymphadenopathy. The KO mice developed hypergammaglobulinemia of all Ig isotypes. Production of anti-nuclear antibodies and anti-double-stranded DNA antibodies was detected in the KO mice. Histological examination showed infiltration of plasma cells in the lung, paraepithelium of the bile duct, and pancreas. Plasma cells also accumulated in the lymph nodes and spleen in the $\mathrm{KO}$ mice. Flow cytometric (FACS) analysis showed that in the case of control mice most B cells were IgDpositive, IgM-negative, and IgD, IgM double-positive. However, for Zc3h12a-deficient B cells, $\sim 70 \%$ of CD19-positive B cells were IgM- and IgD-negative but immunoglobulin-positive, indicating that most Zc3h12adeficient $B$ cells underwent class switching in the spleen of Zc3h12a KO mice. CD138 (Syndecan-1)-positive CD19 dull cells were abundant in the spleen of the KO mice, indicating accumulation of plasma cells in the spleen. When splenic $\mathrm{T}$ cells from the KO mice were stimulated with anti-CD3/CD28 antibody, these T cells produced more IFN- $\gamma$ but less IL-17 compared with control mice, indicating that $\mathrm{T}$ cells in the $\mathrm{KO}$ mice are skewed to Th1 but not Th17 responses. Furthermore, FACS analysis showed that most splenic $\mathrm{T}$ cells in the KO mice were CD62L dull and CD44 high, indicating that they were mostly effector/memory type $\mathrm{T}$ cells. In addition, splenic $\mathrm{T}$ cells from the $\mathrm{KO}$ mice expressed activation marker CD69. The number of FoxP3-positive regulatory T cells (Tregs) was comparable between wild- type and $\mathrm{KO}$ mice. Thus, Zc3h12a is essential for preventing the development of severe immune diseases characterized by an increase in immunoglobulin-producing plasma cells and hyperactivation of $\mathrm{T}$ cells.

When cytokine production from macrophages was examined in response to TLR ligands, production of IL-6 and IL-12p40 but not TNF- $\alpha$ was highly enhanced in response to TLR ligands in the KO mice. We performed microarray analysis to assess the difference in LPS-inducible gene expression in wild-type and Zc3h12a-deficient macrophages. Microarray analysis showed that most LPSinducible genes were comparably expressed in wild-type and Zc3h12a-deficient cells. Nevertheless, a particular set of genes was highly expressed in Zc3h12a-deficient macrophages. They included IL-6, IFN- $\gamma$, and calcitonin receptor, indicating that $\mathrm{Zc} 3 \mathrm{~h} 12 \mathrm{a}$ is responsible for regulation of a limited number of genes in macrophages.

$\mathrm{CCCH}$-type zinc-finger proteins are involved in RNA metabolism pathways such as RNA splicing, polyadenylation, and mRNA decay. Thus, we hypothesized that Zc3h12a might play a role in the destabilization of mRNA. Wild-type and Zc3h12a-deficient macrophages were stimulated with LPS for $2 \mathrm{~h}$ followed by actinomycin D treatment to stop transcription. The half-life of IL-6 mRNA increased in Zc3h12a macrophages compared to wild-type cells, indicating that Zc3h12a regulates IL-6 mRNA post-transcriptionally. However, the TNF or keratinocyte-derived chemokine (KC) mRNA half-life was not altered between wild-type and Zc3h12a-deficient cells, demonstrating that these genes are not posttranscriptionally regulated by Zc3h12a. Mouse IL-6 mRNA contains five AREs and a stem-loop structure in its $3^{\prime}$ UTR; the latter is an $\sim 30$-nucleotide sequence conserved between species and reported to be important for IL-6 mRNA destabilization. To investigate the regions critical for Zc3h12a-dependent destabilization, we generated a series of luciferase reporter constructs containing a variety of regions of IL-6 $3^{\prime}$ UTR, and luciferase activity was measured with and without $\mathrm{Zc} 3 \mathrm{~h} 12 \mathrm{a}$ expression vector. Luciferase activity of reporter constructs containing fragments with AREs only were not altered by Zc3h12a expression, but reporters with fragments containing the stem-loop were decreased, indicating that the stemloop structure appears to be essential for Zc3h12a-mediated decay. In addition, the expression of Zc3h12a reduced the luciferase activity of reporters with $3^{\prime}$ UTRs from IL-12p40 and calcitonin receptor, but not $3^{\prime}$ UTRs from IFN- $\gamma$. Therefore, IL-6, IL-12p40, and calcitonin receptor mRNAs are directly regulated by Zc3h12a.

Sequence alignment showed the presence of a PINdomain-like structure (139-297), just preceding the zinc-finger domain (300-322) (Fig. 3). PIN domains are small protein domains of $\sim 130$ amino acids identified by the presence of three strictly conserved acidic residues (Arcus et al. 2010). PIN-domain proteins are $\mathrm{Mg}^{2+}$-dependent RNases. Of note, recombinant Zc3h12a proteins showed ribonuclease activity in an $\mathrm{Mg}^{2+}$-dependent manner. Mutation of the key amino acid potentially important for enzyme activity (Zc3h12aD141N mutant) did not degrade RNA, indicating that RNase activity is es- 


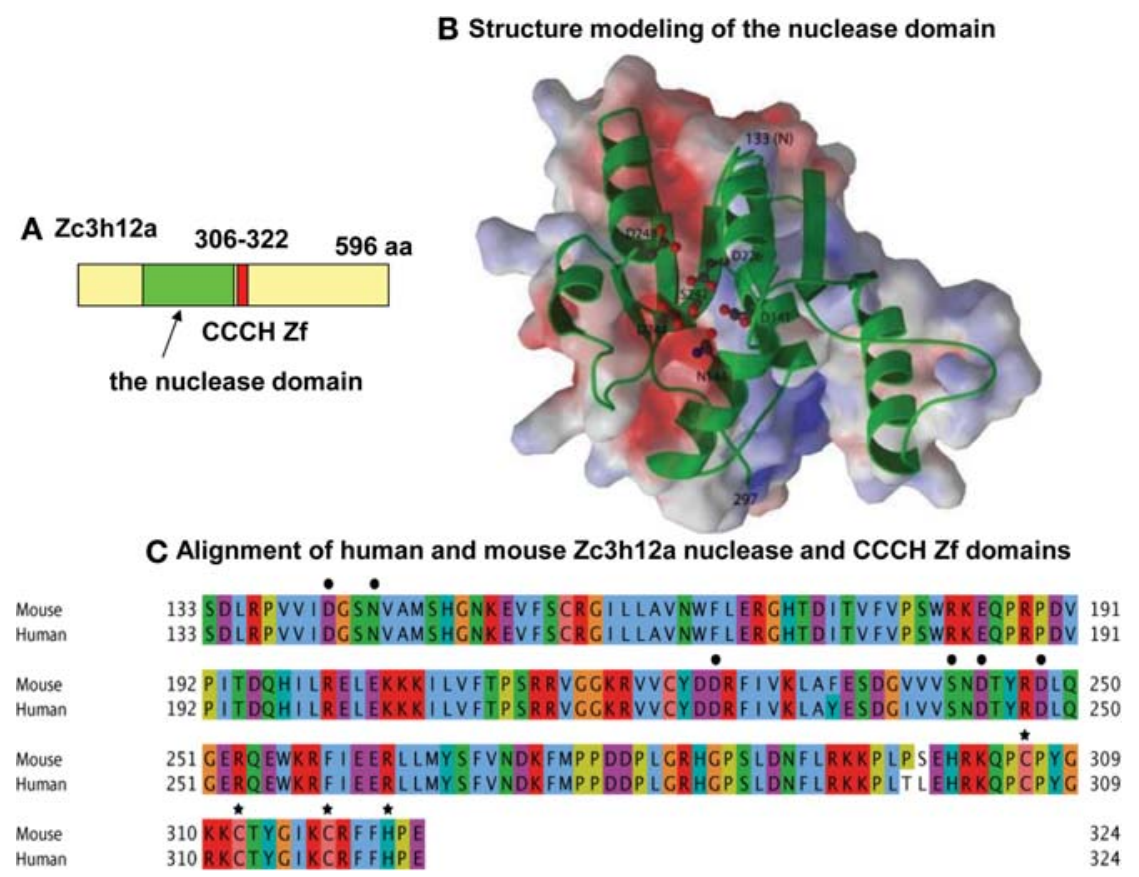

Figure 3. Zc3h12a protein harbors a nuclease domain. (A) Zc3h12a harbors an RNase domain just upstream of the zinc-finger domain (CCCH-type). (B) Structural model of the nuclease domain. (C) Alignment of the nuclease and CCCH domains in mouse and human Zc3h12a.

sential for the function of Zc3h12a. Based on the function we renamed Zc3h12a as Regulatory RNase-1 (Regnase1) (Fig. 4).

\section{MyD88-IKK-DEPENDENT DEGRADATION OF REGNASE-1 PROTEIN}

Although Regnase-1 was identified as an LPS-inducible gene, the protein is present in unstimulated macro- phages. After LPS stimulation, several retarded bands appeared and subsequently all bands disappeared. At the 4-h-time point the bands reappeared, indicating that Regnase- 1 protein was modified and degraded after LPS stimulation, followed by new synthesis of Regnase- 1 protein (Iwasaki et al. 2011). LPS triggers two distinct signaling pathways, MyD88- and TRIF-dependent pathways. Degradation of $\mathrm{I} \kappa \mathrm{B} \alpha$ protein was observed in both MyD88- and TRIF-deficient macrophages. In contrast,

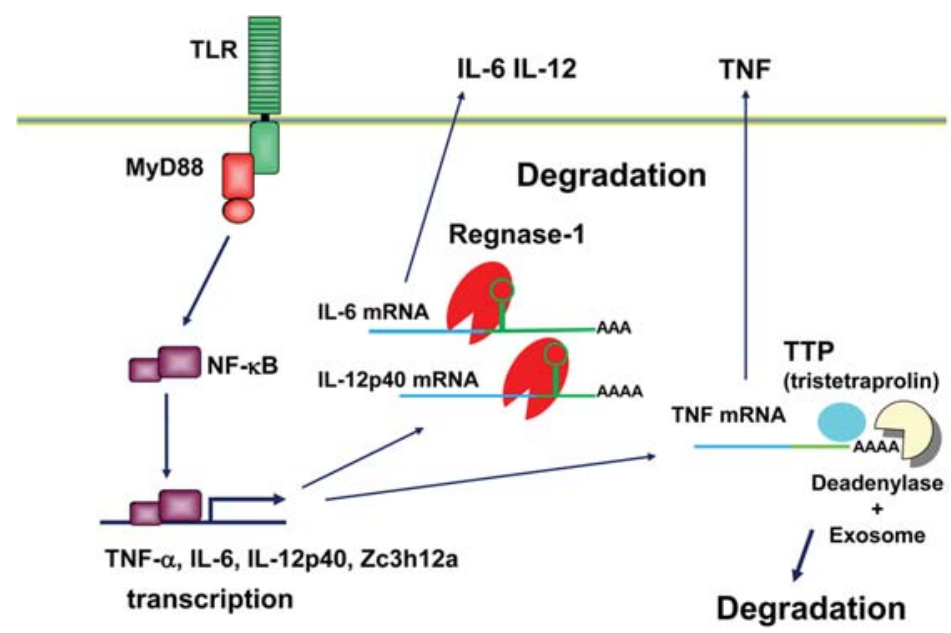

Figure 4. Regnase-1 is an endoribonuclease involved in the regulation of cytokine mRNAs. Zc3h12a mRNA is rapidly induced in macrophages after TLR stimulation and involved in degradation of IL-6 and IL-12p40 mRNAs via binding to the stem-loop structure present in their $3^{\prime}$ UTRs. TTP (tristeraprolin) recruits deadenylases to remove the poly(A) tail, facilitating the subsequent degradation of TNF mRNA. 
in response to LPS Regnase-1 protein disappeared in TRIF-deficient macrophages but not in MyD88-deficient macrophages. Consistent with this finding, TLRs (e.g., TLR2, 4, 7, and 9) that activate MyD88 but not TLR3, which only activate TRIF, degraded Regnase-1. IL-1 $\beta$ stimulation, that activates the MyD88-dependent pathway, also degraded Regnase-1 protein. However, TNF stimulation did not degrade the protein. These results indicate that activation of the MyD88 signaling pathway is required for degradation of Regnase-1. Retardation of Regnase-1 bands was due to phosphorylation because phosphatase treatment abolished the appearance of the retarded bands. IL-1 $\beta$ but not TNF stimulation induced ubiquitination of Regnase-1. A proteasome inhibitor, MG-132, abolished the disappearance of Regnase-1 bands, suggesting phosphorylation-dependent ubiquitinmediated proteasomes are involved in the degradation of Regnase-1. Actinomycin D or cyclohexamide treatment did not impair the disappearance of Regnase-1 bands but impaired the reappearance of Regnase- 1 bands. This indicated the early disappearance of Regnase-1 bands did not require Regnase-1 mRNA or protein synthesis, whereas the bands appearing at the late phase required the induction of Regnase-1 mRNA and subsequent protein synthesis.

We next searched for the kinase responsible for the phosphorylation of Regnase-1. IKKs are known to recognize a consensus motif DSGxxS, and to phosphorylate two serine residues. We found that such a consensus motif was present in the carboxy-terminal region of Regnase-1. Treatment with the IKK- $\beta$ inhibitor TPCA-1 inhibited the degradation of Regnase-1. Cells lacking both IKK- $\alpha$ and IKK- $\beta$ did not show degradation of Reganase- 1 or IкB $\alpha$ in response to LPS. In vitro kinase assays showed recombinant IKK- $\beta$ phosphorylated serine 435 and 439 of recombinant Regnase-1 protein. Coimmunoprecipitation analysis showed that IKK- $\beta$ interacted with Regnase-1 endogenously. It is well known that the phosphorylated DSGxxS sequence is subsequently recognized by the E3 ligase $\beta$-TrCP for polyubiquitination. Coexpression experiments showed the physical interaction of $\beta-\operatorname{TrCP}$ and Regnase-1 in HEK293 human embryonic kidney cells when wild-type IKK- $\beta$ but not kinase-inactive IKK- $\beta$ was coexpressed. Collectively, these results indicate that IKK- $\alpha$,b phosphorylate Regnase- 1 on its canonical DSGxxS sequence and that $\beta-\mathrm{TrCP}$ is involved in the activation of the ubiquitin-proteasome pathway.

We next investigated the mechanism by which Regnase- 1 is degraded by stimulation with IL-1 or TLR ligands but not by TNF. Although the signaling pathways downstream from TAK1 are shared by IL-1R, TLRs, and TNFR, the upstream molecules, MyD88 and IRAKs are unique in IL-1R and TLR signaling. We speculated that modification of Regnase-1 by IRAKs is necessary for its subsequent phosphorylation by IKKs. Cotransfection experiments showed that Regnase- 1 interacted with IRAK1, and was phosphorylated by IRAK-1. Coexpression of IRAK1 augmented the interaction between Regnase- 1 and IKK- $\beta$. Furthermore, coexpression of IRAK1 resulted in augmented ubiquitination of Regnase-1 induced by IKK- $\beta$, suggesting that IRAK1 facilitates Regnase- 1 phosphorylation by IKK- $\beta$ and subsequent ubiquitination by direct interaction and modification of Regnase-1.

Regnase-1 disappears within 15 min after IL-1 or LPS stimulation, and reappears within $2 \mathrm{~h}$ in macrophages. TNF stimulation also induces transcriptional activation of the IL-6 gene. However, TNF stimulation does not degrade Regnase-1. This indicates that the IL-6 mRNA half-life may change during IL-1 or LPS stimulation, but not TNF stimulation. Therefore, we measured IL-6 mRNA half-life at $20 \mathrm{~min}$ and $120 \mathrm{~min}$ after IL-1 or TNF stimulation. We found that IL-1 stimulation extended the half-life of IL-6 mRNA at 20 min in HeLa cells, but TNF did not. Consistent with the reappearance of Regnase-1 protein, the half-life of IL-6 mRNA at 240 min was similar between IL-1 and TNF stimulation, indicating the kinetics of IL-6 mRNA stability correlated with the abundance of Regnase-1 protein in response to IL-1 or TNF.

Regnase-1 3' UTR also has a stem loop structure. When we used the luciferase reporter plasmids with mutations that disrupt stem loop formation, luciferase activity was not affected by Regnase- 1 protein expression. In contrast, when we used a luciferase reporter plasmid in which the stem structure is maintained but the sequence is different from the original, luciferase activity was decreased by Regnase- 1 protein. These results show that the stem-loop structure itself is critical for Regnase-1mediated degradation of Regnase- 1 mRNA. A scenario of the role of Regnase-1 is as follows (Fig. 5): Regnase-1 protein is expressed in resting cells without expression of Regnase-1 mRNA to prevent unwanted production of cytokines. In response to stimulation via TLRs or IL-1R, Regnase-1 protein is phosphorylated by the IKK com-
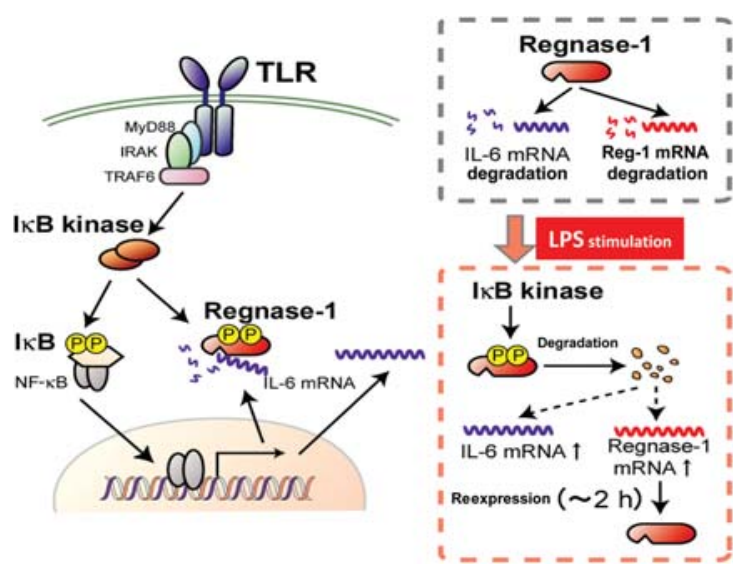

Figure 5. Regnase-1 protein functions as a brake for cytokine production in resting cells. Resting macrophages already transcriptionally activate the IL- 6 gene and produce a small amount of IL- 6 mRNA, which is constantly degraded by Regnase-1 protein. Regnase-1 protein also degrades its own mRNA. Regnase- 1 is rapidly phosphorylated and degraded in response to activation of the IKK complex after LPS stimulation. Thus, expression of Regnase-1 protein in unstimulated cells prevents unwanted production of cytokines. 


\section{REGNASE-1 IN THE REGULATION OF IMMUNE RESPONSES}

plex, which leads to its degradation by an ubiquitin-proteasome-dependent mechanism similar to degradation of I $\mathrm{B}$ proteins. NF- $\kappa \mathrm{B}$ activation induces both IL-6 and Regnase-1 mRNAs. The newly produced Regnase-1 protein suppresses the expression of IL- 6 mRNA as well as Regnase-1 mRNA. The cells return to the initial resting state with expression of Regnase-1 protein alone.

\section{T-CELL-SPECIFIC DELETION OF REGNASE-1}

When we examined the expression of Regnase-1 protein in various tissues, we found that Regnase- 1 protein was highly expressed in immune-related tissues, such as lymph nodes, thymus and spleen. In particular, the thymus expresses the highest amount of Regnase-1 protein. Furthermore, T-cell Regnase-1 protein levels decreased in response to anti-CD3/CD28 antibody stimulation.

To examine the role of Regnase- 1 in T cells we applied the Cre-loxP system. We generated floxed Regnase-1 mice and crossed them with CD4 promoter Cre mice to generate T-cell-specific Regnase-1 KO mice (Uehata et al. 2013). T-cell-specific Regnase-1 KO mice were born alive, but started to die around $8 \mathrm{wk}$ after birth and most died within $17 \mathrm{wk}$. These mice showed severe splenomegaly and lymphadenopathy. Most splenic CD4 and CD8 T cells from conditional $\mathrm{KO}$ mice were $\mathrm{CD}^{2} \mathrm{~L}^{-}$ CD $44^{\text {high }}$ effector/memory T cells. Regnase-1-deficient $\mathrm{T}$ cells also expressed the CD69 activation marker. $\mathrm{CD} 19^{\text {dull }} \mathrm{CD} 138^{\text {high }}$ plasma cells accumulated in the spleen of Regnase-1 KO mice.

Next, we examined thymic T-cell development in T-cell-specific Regnase-1 KO mice. Thymocyte subset populations were not altered. The TCR repertoire of peripheral CD4 $\mathrm{T}$ cells was comparable between $\mathrm{KO}$ mice and control mice. These results suggested that thymic T-cell development was not affected by deletion of Regnase- 1 in T cells.

To investigate whether peripheral CD4 T cells specifically deficient in Regnase-1 are pathogenic, we transferred splenic CD4 T cells from T-cell-specific Regnase-1 $\mathrm{KO}$ mice or control mice into congenic CD45.1 mice. Mice that received Regnase-1-deficient CD4 T cells showed severe splenomegaly, whereas control mice did not show any abnormalities. Regnase-1-deficient CD4 T cells survived and proliferated in recipient mice 3 mo after transfer, although CD4 T cells from control mice were not detected. Furthermore, transfer of Regnase-1-deficient CD4 $\mathrm{T}$ cells alone led to the increase of recipient CD45.1 CD62 ${ }^{\text {low }}$ CD $44^{\text {high }}$ effector/memory $\mathrm{T}$ cells as well as CD19 ${ }^{\text {dull }} \mathrm{CD} 138^{\text {high }}$ plasma cells, showing that Regnase-1 peripheral CD4 T cells can activate other normal immune cells. To further confirm that deletion of Regnase-1 in peripheral CD4 T cells generated pathogenic CD4 $\mathrm{T}$ cells, splenic CD4 $\mathrm{T}$ cells from Mx Cre floxed Regnase-1 mice were transferred into CD45.1 mice, and concurrently treated with poly I:C. Recipient mice showed an increase of CD62 ${ }^{\text {low }}$ CD $44{ }^{\text {high }}$ effector/memory T cells of recipient origin and $\mathrm{CD} 19^{\text {dull }} \mathrm{CD} 138^{\text {high }}$ plasma cells.
These results show Regnase-1 plays a critical role in preventing the aberrant activation of peripheral CD4 T cells.

To examine whether abnormal activation of Regnase-1-deficient CD4 T cells is intrinsic or occurred after antigen stimulation, we generated $\mathrm{OTII}^{+} \mathrm{CD} 4-\mathrm{Cre}$ Regnase-1 floxed mice. OTII TCR transgenic mice express a single TCR specific for a peptide fragment of ovalbumin (OVA). Most CD4 T cells are naïve, indicating that TCR stimulation is required for the development of Regnase-1deficient effector/memory CD4 T cells. We prepared naïve CD4 $\mathrm{T}$ cells from OTII mice lacking Regnase-1 in T cells, and examined the response to TCR stimulation. Compared to wild-type OTII mice, Regnase-1-deficient OTII T cells showed enhanced proliferation. Furthermore, TCR-induced production of effector cytokines such as IFN- $\gamma$, IL-17, and IL-4 was elevated in the absence of Regnase-1. The proportion of FoxP $3{ }^{+}$CD4 Treg cells was comparable between control and Regnase-1deficient CD4 T cells. In addition, Treg cells purified from Mx Cre floxed Regnase-1 mice after poly I:C treatment were competent in suppressing effector $\mathrm{T}$ cells, suggesting that Treg cells are not functionally defective in the absence of Regnase-1.

To understand the molecular basis for pathogenic CD4 T cells lacking Regnase-1, we examined gene expression profiles in CD4 $\mathrm{T}$ cells from conditional $\mathrm{KO}$ mice and control mice. We found many genes were up-regulated in CD4 T cells lacking Regnase-1 including cytokines, surface markers, and transcription factors. To exclude the possibility that the difference in gene expression was simply due to the difference in the proportion of naïve and effector CD4 T cells, we knocked down Regnase-1 expression in Jurkat cells and examined changes in mRNA expression in response to PMA and ionomycin stimulation. Knockdown of Regnase-1 increased expression of c-Rel, Ox40, and IL-2 mRNA after stimulation

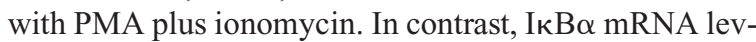
els were comparable between parent and Regnase-1 knockdown Jurkat cells. Reciprocally, overexpression of Regnase-1 in Jurkat cells resulted in the inhibition of the expression of c-Rel, Ox40, and IL-2 mRNA. The expression of Regnase-1 RNase inactive form (D141N) failed to suppress these mRNAs, indicating that the RNase activity of Regnase- 1 is essential for suppressing a set of mRNAs. We previously showed that Regnase- 1 controls mRNA expression via $3^{\prime}$ UTR. To examine whether $3^{\prime}$ UTR is involved in degradation, we generated a set of luciferase reporter constructs with $3^{\prime}$ UTR from a variety of genes involved in T-cell activation. These constructs were transfected into HEK293 cells together with a Regnase-1 expression vector or a nuclease-dead mutant, and luciferase activity was measured. Several target mRNA were identified. They included IL-1 $\beta$, IL-2, ICOS, Ox40, TNFR2, and GATA3. We focused on NF-кB transcription factors. Luciferase assay showed that only c-rel was regulated by Regnase- 1 among the NF-кB subunits. To examine the contribution of c-rel, we generated double $\mathrm{KO}$ mice lacking Regnase-1 and c-rel. In the double $\mathrm{KO}$ mice, the number of effector/memory $\mathrm{T}$ cells was significantly decreased and the number of plasma cells was normal- 
ized, suggesting that c-rel is in part involved in the pathology observed in Regnase-1 KO mice. Furthermore, we found that the level of c-Rel expression was reduced in Rel heterozygous mice compared with wild-type mice, so we examined the effect of decreased c-Rel expression on Regnase-1 KO mice. Decreased c-Rel also resulted in a partial reduction of effector/memory $\mathrm{T}$ cells and plasma cells. These results indicate that increased c-Rel expression contributes to the activation of $\mathrm{T}$ cells and subsequent B-cell activation under Regnase-1 deficiency.

Like macrophages, Regnase-1 protein disappeared in $\mathrm{T}$ cells in response to CD3 plus CD28 or PMA plus ionophore stimulation. However, different from that of macrophages, addition of a proteasome inhibitor did not inhibit the degradation of Regnase-1. Figure 6 shows a comparison between TLR and TCR signaling pathways. Malt1 is reported to act as a protease; therefore, we speculated that Malt 1 may be a candidate for Regnase-1 cleavage. In fact, degradation and cleavage of Regnase-1 were abolished in the presence of a Malt1 protease inhibitor, as well as in Bcl10- or Malt1-deficient CD4 T cells, indicating that Reganse- 1 is cleaved by Malt1. Overexpression of Malt1 expression vector cleaved Regnase-1. We found that R111 of Regnase-1 is a cutting site by using various mutant Regnase-1 expression vectors. Primary CD4 T cells were stimulated with PMA plus ionophore in the presence or absence of Malt1 protease inhibitor, and actinomycin D was added to stop transcription. A set of mRNAs such as c-rel, Ox40, and IL-2 was destabilized in the presence of Malt1 inhibitor, indicating that Regnase- 1 regulated the expression of these mRNAs. In contrast with TCR stimulation, IL-18 stimulation degraded Regnase- 1 in a proteasome-dependent manner similar to macrophages. Therefore, Regnase- 1 degradation is signal-specific but not cell-type-specific.

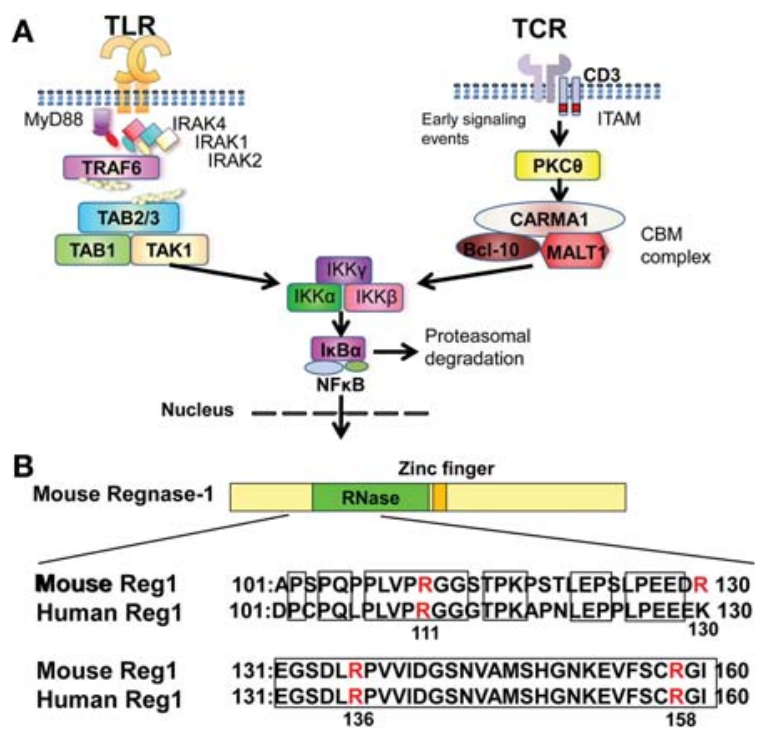

Figure 6. Malt-1 cleaves and degrades Regnase-1 protein. (A) TLR and TCR signaling pathways. Malt 1 protease activity is essential for TCR-mediated Regnase-1 degradation. (B) Regnase- 1 is cleaved by Malt1 at position R111.

\section{CONCLUSION}

Although initial papers described Regnase-1/ Zc3h12a/MIPIP1 as a transcription factor, recent papers confirmed that it has intrinsic RNase activity and is essential for inhibiting the development of lethal autoimmune responses (Matsushita et al. 2009; Mizgalska et al. 2009; Liang et al. 2010; Jura et al. 2012; Xu et al. 2012a, b; Miao et al. 2013; Uehata and Akira 2013). CCCH-type zinc-finger proteins control mRNA decay by associating with the $3^{\prime}$ UTR. For example, TTP and its homologs Zfp3611, Zfp3612, and Zfp3613 are critical for the decay of mRNAs for TNF, GM-CSF, CXCL1, and others (Brooks and Blackshear 2013). Aged TTP KO mice develop autoimmune arthritis because of excessive TNF overproduction (Taylor et al. 1996). Another RING-type ubiquitin ligase protein containing a $\mathrm{CCCH}$ zinc-finger motif called Roquin (also known as Rc3h1) is essential for suppressing autoimmunity by controlling the expression of the ICOS costimulatory molecule (Vinuesa et al. 2005; Yu et al. 2007; Glasmacher et al. 2010). In contrast to TTP and Roquin that require other proteins that catalyze mRNA decay, it is unique that Regnase- 1 acts by itself as an endoribonuclease.

Different from the ARE-binding proteins, Regnase-1 recognizes a stem-loop structure (Matsushita et al. 2009; Iwasaki et al. 2011, Li et al. 2012). We identified several target mRNAs that harbor a stem-loop structure in the $3^{\prime}$ UTR. However, we have not found a rule or consensus sequence from comparison of these stem loop structures. Therefore, we need a comprehensive analysis of target sequences, as well as structural studies on the interaction between Regnase-1 and the stem-loop. Because Regnase-1 does not show sequence specificity in vitro as showed by in vitro RNA cleavage assay, the target specificity may be determined by binding partner(s) of Regnase-1. A recent publication (Leppek et al. 2013) reported that Roquin and Roquin2 specifically recognize a conserved class of stem-loop structures and degrade mRNAs by recruiting the Ccr4-Caf1-Not deadenylase complex. Suzuki et al. (2011) showed that a wide range of premiRNAs are targeted by Regnase- 1 and cleaved at the hairpin structure. Regnase-1 counteracts the ribonuclease Dicer, involved in the cleavage and processing of premiRNA. It is expected that miRNA synthesis is up-regulated, and a set of mRNAs are down-regulated in Regnase1 -deficient cells. The importance of Regnase-1 in miRNA biogenesis should be further investigated. Intriguing is the recent finding that Regnase-1 recognizes viral RNA and possesses potent antiviral activities (Lin et al. 2013), although this requires further convincing data.

We have generated mice lacking other closely related family members, including Zc3h12b, Zc3h12c, and Zc3h12d. They do not show similar abnormalities to Regnase-1 KO mice, indicating the functional difference of other paralogs in vivo (T Satoh and S Akira, unpubl.). Further studies will be needed to clarify the in vivo role of other family members.

Finally, Regnase-1 may be a good drug target for controlling immune responses. Compounds that inhibit 


\section{REGNASE-1 IN THE REGULATION OF IMMUNE RESPONSES}

the degradation of Regnase-1 would be therapeutically useful for chronic inflammation and autoimmune diseases, whereas exploration of small molecules affecting the RNase activity has the potential to enhance adaptive immunity necessary for vaccine development and cancer immunotherapy.

\section{ACKNOWLEDGMENTS}

I thank E. Kamada and M. Kageyama for secretarial assistance, and T. Satoh for figures. This work was supported by the Special Coordination Funds of the Japanese Ministry of Education, Culture, Sports, Science and Technology, and the Ministry of Health, Labour and Welfare in Japan, the Japan Society for the Promotion of Science through the Funding Program for World-Leading Innovative R\&D on Science and Technology (FIRST Program).

\section{REFERENCES}

Anderson P. 2010. Post-transcriptional regulons coordinate the initiation and resolution of inflammation. Nat Rev Immunol 10: $24-35$.

Arcus VL, McKenzie JL, Robson J, Cook GM. 2010. The PINdomain ribonucleases and the prokaryotic VapBC toxin-antitoxin array. Protein Eng Des Sel 24: 33-40.

Bakheet T, Williams BR, Khabar KS. 2006. ARED 3.0: The large and diverse AU-rich transcriptome. Nucleic Acids Res 34: D111-D114.

Beutler B. 2009. Microbe sensing, positive feedback loops, and the pathogenesis of inflammatory diseases. Immunol Rev 227: 248-263.

Brooks SA, Blackshear PJ. 2013. Tristetraprolin (TTP): Interactions with mRNA and proteins, and current thoughts on mechanisms of action. Biochim Biophys Acta 1829: 666-679.

Cheadle C, Fan J, Cho-Chung YS, Werner T, Ray J, Do L, Gorospe M, Becker KG. 2005. Stability regulation of mRNA and the control of gene expression. Ann NY Acad Sci 1058: 196-204.

Glasmacher E, Hoefig KP, Vogel KU, Rath N, Du L, Wolf C, Kremmer E, Wang X, Heissmeyer V. 2010. Roquin binds inducible costimulator mRNA and effectors of mRNA decay to induce microRNA-independent post-transcriptional repression. Nat Immunol 11: 725-733.

Hao S, Baltimore D. 2009. The stability of mRNA influences the temporal order of the induction of genes encoding inflammatory molecules. Nat Immunol 10: 281-288.

Ivanov P, Anderson P. 2013. Post-transcriptional regulatory networks in immunity. Immunol Rev 253: 253-272.

Iwasaki A, Medzhitov R. 2010. Regulation of adaptive immunity by the innate immune system. Science 327: 291-295.

Iwasaki H, Takeuchi O, Teraguchi S, Matsushita K, Uehata T, Kuniyoshi K, Satoh T, Saitoh T, Matsushita M, Standley DM, et al. 2011. The IкB kinase complex regulates the stability of cytokine-encoding mRNA induced by TLR-IL-1R by controlling degradation of regnase-1. Nat Immunol 12: 11671175.

Jura J, Skalniak L, Koj A. 2012. Monocyte chemotactic protein1-induced protein-1 (MCPIP1) is a novel multifunctional modulator of inflammatory reactions. Biochim Biophys Acta 1823: $1905-1913$.

Kawai T, Akira S. 2010. The role of pattern-recognition receptors in innate immunity: Update on Toll-like receptors. Nat Immunol 11: 373-384.

Lemaitre B, Nicolas E, Michaut L, Reichhart JM, Hoffmann JA. 1996. The dorsoventral regulatory gene cassette spätzle/Toll/ cactus controls the potent antifungal response in Drosophila adults. Cell 86: 973-983.

Leppek K, Schott J, Reitter S, Poetz F, Hammond MC, Stoecklin G. 2013. Roquin promotes constitutive mRNA decay via a conserved class of stem-loop recognition motifs. Cell 153: 869-881.

Li M, Cao W, Liu H, Zhang W, Liu X, Cai Z, Guo J, Wang X, Hui Z, Zhang H, et al. 2012. MCPIP1 down-regulates IL-2 expression through an ARE-independent pathway. PLoS One 7: e49841.

Liang J, Song W, Tromp G, Kolattukudy PE, Fu M. 2008a. Genome-wide survey and expression profiling of $\mathrm{CCCH}-$ zinc finger family reveals a functional module in macrophage activation. PLoS One 3: e2880.

Liang J, Wang J, Azfer A, Song W, Tromp G, Kolattukudy PE, $\mathrm{Fu}$ M. 2008b. A novel CCCH-zinc finger protein family regulates proinflammatory activation of macrophages. J Biol Chem 283: 6337-6346.

Liang J, Saad Y, Lei T, Wang J, Qi D, Yang Q, Kolattukudy PE, Fu M. 2010. MCP-induced protein 1 deubiquitinates TRAF proteins and negatively regulates JNK and NF- $\mathrm{KB}$ signaling. J Exp Med 207: 2959-2973.

Lin RJ, Chien HL, Lin SY, Chang BL, Yu HP, Tang WC, Lin YL. 2013. MCPIP1 ribonuclease exhibits broad-spectrum antiviral effects through viral RNA binding and degradation. Nucleic Acids Res 41: 3314-3326.

Matsushita K, Takeuchi O, Standley DM, Kumagai Y, Kawagoe T, Miyake T, Satoh T, Kato H, Tsujimura T, Nakamura H, et al. 2009. Zc3h12a is an RNase essential for controlling immune responses by regulating mRNA decay. Nature 458: 1185-1190.

Miao R, Huang S, Zhou Z, Quinn T, Van Treeck B, Nayyar T, Dim D, Jiang Z, Papasian CJ, Eugene Chen Y, et al. 2013. Targeted disruption of MCPIP1/Zc3h12a results in fatal inflammatory disease. Immunol Cell Biol 91: 368-376.

Mizgalska D, Wegrzyn P, Murzyn K, Kasza A, Koj A, Jura J, Jarzab B. 2009. Interleukin-1-inducible MCPIP protein has structural and functional properties of RNase and participates in degradation of IL-1 $\beta$ mRNA. FEBS J 276: 73867399.

Niu J, Azfer A, Zhelyabovska O, Fatma S, Kolattukudy PE. 2008. Monocyte chemotactic protein (MCP)-1 promotes angiogenesis via a novel transcription factor, MCP-1-induced protein (MCPIP). J Biol Chem 283: 14542-14551.

O'Neill LA, Bowie AG. 2007. The family of five: TIR-domaincontaining adaptors in Toll-like receptor signalling. Nat Rev Immunol 7: 353-364.

Schoenberg DR. 2011. Mechanisms of endonuclease-mediated mRNA decay. Wiley Interdiscip Rev RNA 2: 582-600.

Schoenberg DR, Maquat LE. 2012. Regulation of cytoplasmic mRNA decay. Nat Rev Genet 13: 246-259.

Shaw G, Kamen R. 1986. A conserved AU sequence from the $3^{\prime}$ untranslated region of GM-CSF mRNA mediates selective mRNA degradation. Cell 46: 659-667.

Suzuki HI, Arase M, Matsuyam H, Choi YL, Ueno T, Mano H, Sugimoto K, Miyazono K. 2011. MCPIP1 ribonuclease antagonizes dicer and terminates microRNA biogenesis through precursor microRNA degradation. Mol Cell 44: 424-436.

Takeuchi O, Akira S. 2010. Pattern recognition receptors and inflammation. Cell 140: 805-820.

Taylor GA, Carballo E, Lee DM, Lai WS, Thompson MJ, Patel DD, Schenkman DI, Gilkeson GS, Broxmeyer HE, Haynes $\mathrm{BF}$, et al. 1996. A pathogenetic role for TNF $\alpha$ in the syndrome of cachexia, arthritis, and autoimmunity resulting from tristetraprolin (TTP) deficiency. Immunity 4: 445-454.

Uehata T, Akira S. 2013. mRNA degradation by the endoribonuclease Regnase-1/ZC3H12a/MCPIP-1. Biochim Biophys Acta 1829: 708-713.

Uehata T, Iwasaki H, Vandenbon A, Matsushita K, HernandezCuellar E, Kuniyoshi K, Satoh T, Mino T, Suzuki Y, Standley $\mathrm{DM}$, et al. 2013. Malt1-induced cleavage of regnase-1 in $\mathrm{CD}^{+}$helper $\mathrm{T}$ cells regulates immune activation. Cell 153: 1036-1049. 
Vinuesa CG, Cook MC, Angelucci C, Athanasopoulos V, Rui L, Hill KM, Yu D, Domaschenz H, Whittle B, Lambe T, et al. 2005. A RING-type ubiquitin ligase family member required to repress follicular helper $\mathrm{T}$ cells and autoimmunity. Nature 435: $452-458$

Xu J, Fu S, Peng W, Rao Z. 2012a. MCP-1-induced protein-1, an immune regulator. Protein Cell 3: 903-910.

Xu J, Peng W, Sun Y, Wang X, Xu Y, Li X, Gao G, Rao Z. 2012b. Structural study of MCPIP1 N-terminal conserved domain reveals a PIN-like RNase. Nucleic Acids Res 40: 6957-6965.
Yu D, Tan AH, Hu X, Athanasopoulos V, Simpson N, Silva DG, Hutloff A, Giles KM, Leedman PJ, Lam KP, et al. 2007. Roquin represses autoimmunity by limiting inducible T-cell co-stimulator messenger RNA. Nature 450: 299303.

Zhou L, Azfer A, Niu J, Graham S, Choudhury M, Adamski FM, Younce C, Binkley PF, Kolattukudy PE. 2006. Monocyte chemoattractant protein-1 induces a novel transcription factor that causes cardiac myocyte apoptosis and ventricular dysfunction. Circ Res 98: 1177-1185. 


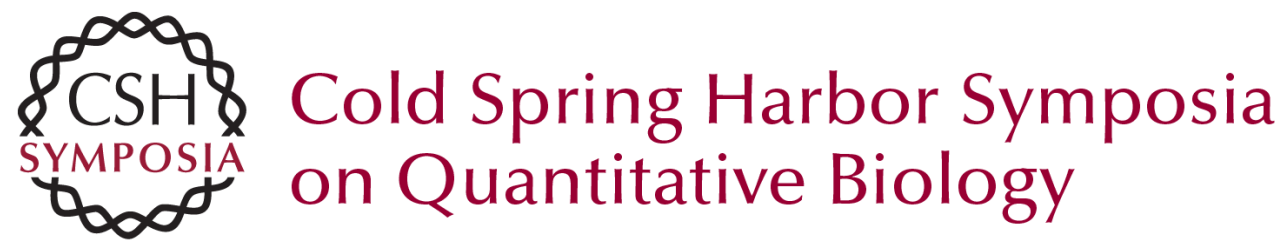

\section{Regnase-1, a Ribonuclease Involved in the Regulation of Immune Responses}

Shizuo Akira

Cold Spring Harb Symp Quant Biol 2013 78: 51-60 originally published online October 25, 2013 Access the most recent version at doi:10.1101/sqb.2013.78.019877

References This article cites 38 articles, 5 of which can be accessed free at: http://symposium.cshlp.org/content/78/51.full.html\#ref-list-1

License

Email Alerting Receive free email alerts when new articles cite this article - sign up in Service the box at the top right corner of the article or click here. 